\title{
ON UNIFORM DISTRIBUTION AND THE DENSITY OF SUM SETS
}

\author{
BODO VOLKMANN
}

1. For any lattice point $\mathfrak{a}=\left(a_{1}, \cdots, a_{k}\right)$ in the $k$-dimensional euclidean space $R^{k}$, let $\|\mathfrak{a}\|=\max _{k=1, \cdots, k}\left|a_{k}\right|$. If $A$ is an infinite set of such lattice points, we define for $x>0$ the counting function $A(x)$ to be the number of elements $\mathfrak{a} \in A$ satisfying $\|\mathfrak{a}\| \leqq x$. Then various densities for such sets $A$ can be introduced as generalizations of the well-known densities of sets of non-negative integers. In particular, we shall denote the lower limit, the upper limit, and, in the case of its existence, the limit, of the sequence $A(x) /(2 x)^{k}$, as $x$ tends to infinity, by $d_{*}(A), d^{*}(A)$, and $d(A)$, respectively. Furthermore, if $A$ is restricted to have elements with non-negative coordinates only, we shall consider the corresponding expressions of the sequence $A(x) / x^{k}$ and denote them by $D_{*}(A), D^{*}(A)$, and $D(A)$. According to the terminology in the case $k=1$, we shall call these limits the lower and upper asymptotic densities and the natural density ${ }^{1}$ of $A$, respectively.

The sum set $A+B$ of two sets $A$ and $B$ in $R^{k}$ is, as usual, defined to be the set of all points $\mathfrak{a}+\mathfrak{b}, \mathfrak{a} \in A, \mathfrak{b} \in B$, obtained by vector addition.

By an interval $I \subseteq R^{k}$ we mean the cartesian product of any $k$ open intervals of $R^{1}$. The unit cube, i.e. the set of all points $x=\left(x_{1}, \cdots, x_{k}\right)$ with $0 \leqq x_{k}<1(\kappa=1, \cdots, k)$, is denoted by $C^{k}$.

For any real number $x$, let $[x]$ denote the greatest integer $\leqq x$ and let $\{x\}$ be the fractional part $x-[x]$. Then, for every point $\mathfrak{x}=\left(x_{1}, \cdots, x_{k}\right)$, let $\{\mathfrak{x}\}=\left(\left\{x_{1}\right\}, \cdots,\left\{x_{k}\right\}\right)$, and for every set $M \subseteq R^{k}$, let $\{M\}$ be the set of all points $\{\mathfrak{x}\}$ with $\mathfrak{r} \in M$.

The Jordan content of a set $M \subseteq R^{k}$ is denoted by $\mu_{k}(M)$.

A sequence $\mathfrak{x}_{1}, \mathfrak{x}_{2}, \cdots$ is called uniformly distributed $(\bmod 1)$ if it has the following property: Let, for any interval $I \subseteq C^{k}, N_{I}$ be the set of indices $i$ such that $\left\{\mathfrak{x}_{i}\right\} \in I$. Then $N_{I}$ has a natural density $D\left(N_{I}\right)=\mu_{k}(I)$.

2. In the case $k=1$ an analogue of Mann's Theorem has been proved for lower asymptotic densities by M. Kneser [3]. In the absence of a similar theorem for $k>1$ the present paper aims at establishing the inequality of the $(\alpha+\beta)$-theorem for a certain class of sets

Received by the editors March 12, 1956.

${ }^{1}$ See, for instance, B. Volkmann [6]. 
of lattice points defined by means of uniformly distributed sequences.

Let $\lambda_{1}, \cdots, \lambda_{k}$ be fixed positive irrational numbers and let, for any lattice point $\mathfrak{a}=\left(a_{1}, \cdots, a_{k}\right), \mathfrak{p}(\mathfrak{a})=\left(\lambda_{1} a_{1}, \cdots, \lambda_{k} a_{k}\right)$. Furthermore, if $M$ is an open subset of $C^{k}$, let $A_{M}$ be the set of lattice points a such that $\{\mathfrak{p}(\mathfrak{a})\} \in M$. If then $A_{M}^{+}$denotes the set of those elements of $A_{M}$ which have non-negative coordinates only, the following theorem holds:

TheOREM 1. For any two open sets $M_{1} \subseteq C^{k}$ and $M_{2} \subseteq C^{k}$, the following densities exist and satisfy the inequalities:

$$
D\left(A_{\left.M_{M_{1}+M_{2}}\right\}}^{+}\right) \geqq \min \left(1, D\left(A_{M_{1}}^{+}\right)+D\left(A_{M_{2}}^{+}\right)\right)
$$

and

$$
d\left(A_{\left(M_{1}+M_{2}\right\}}\right) \geqq \min \left(1, d\left(A_{M_{1}}\right)+d\left(A_{M_{2}}\right)\right) .
$$

For the proof the following lemmas are needed:

LEMma 1. Under the conditions of the theorem,

$$
A_{\left\{M_{1}+M_{2}\right\}}^{+} \supseteq A_{M_{1}}^{+}+A_{M_{2}}^{+} \text {. }
$$

Proof. Let $\mathfrak{a}$ be an element of $A_{M_{1}}^{+}+A_{M_{2}}^{+}$. Then there are lattice points $\mathfrak{a}_{1}$ and $\mathfrak{a}_{2}$ with non-negative coordinates such that $\mathfrak{a}=\mathfrak{a}_{1}+\mathfrak{a}_{2}$, $\left\{\mathfrak{p}\left(\mathfrak{a}_{1}\right)\right\} \in M_{1},\left\{\mathfrak{p}\left(\mathfrak{a}_{2}\right)\right\} \in M_{2}$. Hence $\left\{\mathfrak{p}\left(\mathfrak{a}_{1}+\mathfrak{a}_{2}\right)\right\} \in\left\{M_{1}+M_{2}\right\}$ and consequently, $\mathfrak{a} \in A_{\left\{M_{1}+M_{2}\right\}}^{+}$.

LEMma 2. Under the conditions of the theorem,

$$
A_{\left\{M_{1}+M_{2}\right\}}=A_{M_{1}}+A_{M_{2}} .
$$

Proof. As in Lemma 1, the relation

$$
A_{\left\{M_{1}+M_{2}\right\}} \supseteq A_{M_{1}}+A_{M_{2}}
$$

is obtained immediately. To prove the opposite inclusion, let $\mathfrak{a} \in A_{\left\{M_{1}+M_{2}\right\}}$. Then $\{\mathfrak{p}(\mathfrak{a})\} \in\left\{M_{1}+M_{2}\right\}$, i.e.

$$
\{\mathfrak{p}(\mathfrak{a})\} \equiv \mathfrak{m}_{1}+\mathfrak{m}_{2}(\bmod 1), \quad \mathfrak{m}_{1} \in M_{1}, \mathfrak{m}_{2} \in M_{2} .
$$

Since the sets $M_{1}$ and $M_{2}$ are open there exists an $\epsilon>0$ such that the interval

$$
\begin{array}{r}
\left(m_{i 1}-\epsilon, m_{i 1}+\epsilon\right) \times\left(m_{i 2}-\epsilon, m_{i 2}+\epsilon\right) \times \cdots \times\left(m_{i k}-\epsilon, m_{i k}+\epsilon\right) \\
(i=1,2)
\end{array}
$$

is contained in $M_{i}$ if $\mathfrak{m}_{i}=\left(m_{i 1}, \cdots, m_{i k}\right)$. By a theorem due to Hermann Weyl [7] each of the $k$ sequences $\lambda_{x} a(a=0,1,2, \cdots)$ is uni- 
formly distributed and therefore the sequences $\left\{\lambda_{\kappa} a\right\}$ are everywhere dense in the interval $C^{1}$. Thus there exists a lattice point $\mathfrak{a}_{1}=\left(a_{11}, \cdots, a_{1 k}\right)$ such that

$$
m_{1 \kappa}-\epsilon<\left\{\lambda_{\kappa} a_{1 \kappa}\right\}<m_{1 \kappa}+\epsilon \quad \quad(\kappa=1, \cdots
$$

and consequently, $\mathfrak{a}_{1} \in A_{M_{1}}$. Letting $\mathfrak{a}_{2}=\mathfrak{a}-\mathfrak{a}_{1}$ one obtains

$$
\{\mathfrak{p}(\mathfrak{a})\}-\left\{\mathfrak{p}\left(\mathfrak{a}_{1}\right)\right\} \equiv\left\{\mathfrak{p}\left(\mathfrak{a}_{2}\right)\right\}(\bmod 1)
$$

and hence, if $\mathfrak{a}_{2}=\left(a_{21}, \cdots, a_{2 k}\right),(7)$ and (6) imply

$$
\left\{p\left(\mathfrak{a}_{2}\right)\right\} \in\left(m_{21}-\epsilon, m_{2 l}+\epsilon\right) \times \cdots \times\left(m_{2 k}-\epsilon, m_{2 k}+\epsilon\right),
$$

therefore $\mathfrak{a}_{2} \in A_{M_{2}}$ and $\mathfrak{a} \in A_{M_{1}}+A_{M_{2}}$. In view of (5), this establishes the lemma.

LeMmA 3. If the set of all lattice points in $R^{k}$ is ordered as a sequence $\mathfrak{a}_{1}, \mathfrak{a}_{2}, \ldots$ in such a way that $\left\|\mathfrak{a}_{m}\right\|<\left\|\mathfrak{a}_{n}\right\|$ implies $m<n$, then the sequence $\mathfrak{p}\left(\mathfrak{a}_{1}\right), \mathfrak{p}\left(\mathfrak{a}_{2}\right), \cdots$ is uniformly distributed.

Proof. From Weyl's theorem referred to above, it follows that each of the $k$ sequences $\lambda_{k} a(a=0, \pm 1, \pm 2, \cdots)$ is uniformly distributed in the sense that, for any interval $I_{k} \subseteq C^{1}$, the set $A_{I_{\kappa}}$ has the density $d\left(A_{I_{k}}\right)=\mu_{1}\left(I_{\kappa}\right)$. By definition, the first $(2 x+1)^{k}$ terms of the sequence $\mathfrak{a}_{1}, \mathfrak{a}_{2}, \ldots$ are exactly all the lattice points $\mathfrak{a}$ with $\|\mathfrak{a}\| \leqq x$. Therefore, if $I=I_{1} \times I_{2} \times \cdots \times I_{k}$ is an interval in $C^{k}$, then the counting function of the set $A_{I}$ is

$$
A_{I}(x)=A_{I_{1}}(x) A_{I_{2}}(x) \cdots A_{I_{k}}(x),
$$

and thus the $k$ asymptotic equations

$$
A_{I \kappa}(x) \simeq 2 x \cdot \mu_{1}\left(I_{\kappa}\right) \quad(\kappa=1, \cdots, k)
$$

imply

$$
A_{I}(x) \simeq(2 x)^{k} \prod_{k=1}^{k} \mu_{1}\left(I_{\kappa}\right)=(2 x)^{k} \mu_{k}(I)
$$

and therefore $d\left(A_{I}\right)=\mu_{k}(I)$.

Since obviously $D\left(A_{I_{\kappa}}^{+}\right)=d\left(A_{I_{\kappa}}\right)$, one also obtains

$$
D\left(A_{I}^{+}\right)=\mu_{k}(I) \text {. }
$$

Lemma 4. For any open set $M \subseteq C^{k}$ there exist the densities

$$
d\left(A_{M}\right)=D\left(A_{M}^{+}\right)=\mu_{k}(M) .
$$

Proof. Let $\epsilon>0$, then there are sets $R_{\epsilon}$ and $R^{\epsilon}$ which are finite 
unions of intervals, such that $R_{\epsilon} \subseteq M \subseteq R^{\epsilon}$ and $\mu_{k}\left(R^{\epsilon}\right)-\mu_{k}\left(R_{\epsilon}\right)<\epsilon$. Then Lemma 3 implies that

$$
\mu_{k}\left(R_{\epsilon}\right) \leqq d_{*}\left(A_{M}\right) \leqq d^{*}\left(A_{M}\right) \leqq \mu_{k}\left(R^{\epsilon}\right)
$$

and therefore, since $\epsilon$ is arbitrary, $d\left(A_{M}\right)=\mu_{k}(M)$. The equation $D\left(A_{M}^{+}\right)=\mu_{k}(M)$ is obtained analogously.

Lemma 5. For any two open sets $M_{1} \subseteq C^{k}, M_{2} \subseteq C^{k}$,

$$
\mu_{k}\left(\left\{M_{1}+M_{2}\right\}\right) \geqq \min \left(1, \mu_{k}\left(M_{1}\right)+\mu_{k}\left(M_{2}\right)\right) .
$$

Proof. If all boundary points of $C^{k}$ which the set $\left\{M_{1}+M_{2}\right\}$ may contain are removed from it, the remaining set is obviously open. Therefore $\left\{M_{1}+M_{2}\right\}$ has a content and (8) follows directly from A. M. Macbeath [5, Theorem 1].

Now Theorem 1 follows from Lemma 4, applied to the three sets $M_{1}, M_{2}$, and $\left\{M_{1}+M_{2}\right\}$ and Lemma 5 .

Corollary. If $M_{1}, \cdots, M_{n}$ are open subsets of $C^{1}$, then

$$
A\left\{M_{2}+\cdots+M_{n}\right\}=A_{M_{1}}+\cdots+A_{M_{n}}
$$

and

$$
d\left(A\left\{M_{1}+\cdots+M_{n}\right\}\right) \geqq \min \left(1, \sum_{i=1}^{n} d\left(A_{M_{i}}\right)\right) .
$$

Proof. Follows from the theorem by induction.

3. In the case $k=1$, Theorem 1 can be proved directly from Kneser's Theorem mentioned above, and consequently, the special case for linear, open sets of Macbeath's Theorem follows then as a corollary.

To establish this, we use the concept of a rational set of non-negative integers, ${ }^{2}$ i.e. a set whose characteristic function with respect to the set of all non-negative integers is ultimately periodic. In this sense the set $A_{M_{1}}^{+}+A_{M_{2}}^{+}$is not rational whenever its lower asymptotic density is different from 1 , for otherwise there would be some residue class $P$ such that the intersection $P^{+} \cap\left(A_{M_{1}}^{+}+A_{M_{2}}^{+}\right)$is empty or finite. If then $P_{1}$ and $P_{2}$ are any two residue classes such that $P_{1}^{+}+P_{2}^{+}=P^{+}$, it follows that at least one of the intersections $A_{M_{1}}^{+} \cap P_{1}^{+}$and $A_{M_{2}}^{+} \cap P_{2}^{+}$, say, the first one, is empty or finite; otherwise $A_{M_{1}}^{+}+A_{M_{2}}^{+}$would contain infinitely many elements of $P^{+}$. But the sequence $\left\{\lambda_{1} a\right\}$ with $a \in P_{1}^{+}$is itself uniformly distributed ${ }^{3}$ and must therefore, because of

${ }^{2}$ Cf. [1] and [6].

${ }^{3}$ Cf. [7]. 
$\mu_{1}\left(M_{1}\right)>0$, have infinitely many elements in $M_{1}$. This contradicts the assumption that $A_{M_{1}}^{+}+A_{M_{2}}^{+}$is rational.

Kneser's Theorem implies that for any two sets $A$ and $B$ of nonnegative integers whose sum set $A+B$ is not rational,

$$
D_{*}(A+B) \geqq \min \left(1, D_{*}(A)+D_{*}(B)\right) \text {. }
$$

This proves (1) from which (2) can easily be obtained by decomposing the sets $A_{M_{1}}$ and $A_{M_{2}}$ into the subsets of their non-negative and of their negative elements.

4. The question may be raised what values the density $d\left(A_{1}+\cdots+A_{n}\right)$ can assume if $d\left(A_{1}\right), \cdots, d\left(A_{n}\right)$ are prescribed. As an answer to this question we prove the following

THEOREM $2 .^{4}$ If $\alpha_{1}, \cdots, \alpha_{n}$, and $\gamma$ are positive real numbers satisfying $\sum_{i=1}^{n} \alpha_{i} \leqq \gamma \leqq 1$, then there are sets $A_{1}, \cdots, A_{n}$ of lattice points in $R^{k}$ such that

$$
d\left(A_{i}\right)=\alpha_{i}(i=1, \cdots, n) \text { and } d\left(A_{1}+\cdots+A_{n}\right)=\gamma .
$$

Proof. In view of Lemmas 2 and 4 it suffices to show that there are open subsets $M_{1}, \cdots, M_{n}$ of $C^{k}$ such that

$$
\mu_{k}\left(M_{i}\right)=\alpha_{i}(i=1, \cdots, n) \text { and } \mu_{k}\left(\left\{M_{1}+\cdots+M_{n}\right\}\right)=\gamma,
$$

since the conditions of the theorem are then satisfied by the sets

$$
A_{i}=A_{M_{i}}, \quad A_{1}+\cdots+A_{n}=A_{\left\{M_{1}+\cdots+M_{n}\right\}} .
$$

Furthermore, we may restrict the proof to the case $k=1$; for, if $M_{1}, \cdots, M_{n}$ are subsets of $C^{1}$ satisfying (11), then the cartesian products $M_{1} \times \bar{C}^{k-1}, \cdots, M_{n} \times \bar{C}^{k-1}$ together with the set $\left\{\left(M_{1} \times \bar{C}^{k-1}\right)+\cdots+\left(M_{n} \times \bar{C}^{k-1}\right)\right\}=\left\{M_{1}+\cdots+M_{n}\right\} \times \bar{C}^{k-1}$, $\bar{C}^{k-1}$ being the interior of $C^{k-1}$, will satisfy (11) in the $k$-dimensional sense.

Such sets $M_{i}$ can, for example, be constructed as follows: It may be assumed without loss of generality that $\alpha_{1} \geqq \alpha_{2} \geqq \cdots \geqq \alpha_{n}$. Then, for $i=1, \cdots, n-1$, let $M_{i}$ be the open interval $\left(0, \alpha_{i}\right)$ and let $\sigma=\sum_{i=1}^{n-1} \alpha_{i}$. For the definition of $M_{n}$ the following two cases are distinguished:

(a) If $\gamma / \sigma$ is an integer $q$ (hence $q \geqq 2$ since $\gamma-\sigma \geqq \alpha_{n}>0$ ), let

$$
M_{n}=\bigcup_{j=0}^{q-2}\left(j \sigma, j \sigma+\frac{\alpha_{n}}{q}\right) \cup\left((q-1) \sigma-\frac{\alpha_{n}}{q},(q-1) \sigma\right) .
$$

4 In the case of lower asymptotic densities of sets of integers a similar existence theorem was proved, by a different method, by L. P. Cheo [2] who used an idea of B. Lepson [4]. 
These $q$ intervals are nonoverlapping as the distance of the "last" two is $\sigma-2 \alpha_{n} / q=\sigma\left(1-2 \alpha_{n} / \gamma\right)>0$ (for $\gamma \geqq \sum_{i=1}^{n} \alpha_{i} \geqq 2 \alpha_{n}$ ), and otherwise the distance of any two adjacent intervals is $\sigma-\alpha_{n} / q>0$. Therefore, $\mu_{1}\left(M_{n}\right)=\alpha_{n}$ and, as is readily seen, $M_{1}+\cdots+M_{n-1}=(0, \sigma)$, consequently

$$
\begin{aligned}
\left\{M_{1}+\cdots+M_{n}\right\}= & M_{1}+\cdots+M_{n}=\bigcup_{j=0}^{q-2}\left(j \sigma,(j+1) \sigma+\frac{\alpha_{n}}{q}\right) \\
& \cup\left((q-1) \sigma-\frac{\alpha_{n}}{q}, q \sigma\right)=(0, q \sigma)=(0, \gamma) .
\end{aligned}
$$

(b) If $\gamma / \sigma$ is not an integer, a number $\epsilon>0$ is to be chosen such that

$$
\epsilon<\min \left(\frac{\alpha_{n}}{\lfloor\gamma / \sigma]}, \frac{1}{2}(\gamma-[\gamma / \sigma] \sigma)\right)
$$

and $M_{n}$ is defined as

$$
M_{n}=\left(0, \alpha_{n}-\epsilon[\gamma / \sigma]\right) \cup\left(\bigcup_{j=1}^{[\gamma / \sigma-1]}(j \sigma, j \sigma+\epsilon)\right) \cup(\gamma-\sigma-\epsilon, \gamma-\sigma) .
$$

Then as in case (a) the intervals of $M_{n}$ are nonoverlapping as the "first" two of them obviously have a positive distance, the "last" two of them have the distance

$$
(\gamma-\sigma-\epsilon)-\left(\left[\frac{\gamma}{\sigma}\right]-\sigma+\epsilon\right)=\gamma-\left[\frac{\gamma}{\sigma}\right] \sigma-2 \epsilon>0
$$

and otherwise the distance between any two neighboring intervals is

$$
\sigma-\epsilon>\sigma-\frac{\alpha_{n}}{[\gamma / \sigma]}>\sigma\left(1-\frac{\alpha_{n}}{\gamma}\right)>0 .
$$

Thus

$$
\mu_{1}\left(M_{n}\right)=\alpha_{n}-\epsilon\left[\frac{\gamma}{\sigma}\right]+\epsilon \cdot\left[\frac{\gamma}{\sigma}-1\right]+\epsilon=\alpha_{n}
$$

and

$$
\left\{M_{1}+\cdots+M_{n}\right\}=(0, \sigma)+M_{n}=(0, \gamma) .
$$

Added in proof (January 11, 1957). The inequalities of Theorem 1 may be expressed as 


$$
\begin{aligned}
D\left(A_{M_{1}}^{+}+A_{M_{2}}^{+}\right) & \geqq \min \left(1, D\left(A_{M_{1}}^{+}\right)+D\left(A_{M_{2}}^{+}\right)\right), \\
d\left(A_{M_{1}}+A_{M_{2}}\right) & \geqq \min \left(1, d\left(A_{M_{1}}\right)+d\left(A_{M_{2}}\right)\right)
\end{aligned}
$$

in view of Lemma 2 and the following

Lemma 6. $D\left(A_{\left\{M_{1}+M_{2}\right\}}^{+}\right)=D\left(A_{M_{1}}^{+}+A_{M_{2}}^{+}\right)$.

Proof. Consider a covering of the set $M_{1}$ by a finite number of cubes $C_{i}$ with $\mu_{k}\left(C_{i}\right)=\epsilon$, and let $S$ be the set of points $\mathfrak{m}$ in $\left\{M_{1}+M_{2}\right\}$ which have representations

$$
\mathfrak{m} \equiv \mathfrak{m}_{1}+\mathfrak{m}_{2}(\bmod 1),
$$$$
\mathfrak{m}_{i} \in M_{i},
$$

for at least all the points $m_{1}$ in one of the $C_{i}$ 's. Then the remaining set $R(\epsilon)=\left\{M_{1}+M_{2}\right\}-S$ satisfies $\lim _{\epsilon \rightarrow 0} \mu_{k}(R(\epsilon))=0$. Due to uniform distribution, there is an $N(\boldsymbol{\epsilon})$ such that each $C_{i}$ contains a point $\mathfrak{g}\left(\mathfrak{a}_{1}\right), \mathfrak{a}_{1} \in A_{M_{1}}^{+}$, with $\left\|\mathfrak{a}_{1}\right\| \leqq N(\epsilon)$. Thus, if $\mathfrak{a}=\left(a_{1}, \cdots, a_{k}\right) \in A_{S}^{+}$and $a_{\kappa} \geqq N(\epsilon)$ for $\kappa=1, \cdots, k$, then there is such a point $\mathfrak{a}_{1}$ for which $\left\{\mathfrak{g}(\mathfrak{a})-\mathfrak{g}\left(\mathfrak{a}_{1}\right)\right\} \in M_{2}$ and, since all coordinates of $\mathfrak{a}-\mathfrak{a}_{1}$ are non-negative, $\mathfrak{a}-\mathfrak{a}_{1} \in A_{M_{2}}^{+}$. Therefore, $A_{S}^{+} \subseteq A_{M_{1}}^{+}+A_{M_{2}}^{+}$, hence

$$
D\left(A_{S}^{+}\right)=D\left(A_{\left\{M_{1}+M_{2}\right\}}^{+}\right)-\mu_{k}(R(\epsilon)) \subseteq D\left(A_{M_{1}}^{+}+A_{M_{2}}^{+}\right) .
$$

This proves the contention by virtue of Lemma 1 .

Theorem 2 is also true for natural densities $D(A)$ of sets of lattice points with non-negative coordinates; in the proof reference has to be made to Lemma 6 instead of to Lemma 2 .

\section{REFERENCES}

1. R. C. Buck, The measure-theoretic approach to density, Amer. J. Math. vol. 68 (1946) pp. 560-580.

2. L. P. Cheo, $A$ remark on the $\alpha+\beta$-theorem, Proc. Amer. Math. Soc. vol. 3 (1952) pp. 175-177.

3. M. Kneser, Abschätzung der asymptotischen Dichte von Summenmengen, Math. Zeit. vol. 58 (1953) pp. 459-484.

4. B. Lepson, Certain best results in the theory of Schnirelmann density, Proc. Amer. Math. Soc. vol. 1 (1950) pp. 592-594.

5. A. M. Macbeath, On measure of sum sets. II. The sum theorem for the torus, Proc. Cambridge Philos. Soc. vol. 49 (1953) pp. 40-43.

6. B. Volkmann, Über Klassen von Mengen natürlicher Zahlen, J. Reine Angew. Math. vol. 190 (1952) pp. 199-230.

7. H. Weyl, Über die Gleichverteilung von Zahlen mod 1, Math. Ann. vol. 77 (1916) pp. 313-352.

\section{UNIVERSITY OF UTAH AND \\ UNIVERSITY OF MAINZ}

